\title{
XPR1 mutations are a rare cause of primary familial brain calcification
}

\author{
Mathieu Anheim ${ }^{1,2,3}$ - Uriel López-Sánchez ${ }^{4,5,6,7} \cdot$ Donatella Giovannini, 4,,6,7 \\ Anne-Claire Richard ${ }^{8,9,10}$ - Jawida Touhami ${ }^{4,5,6,7}$ - Ludovic N'Guyen ${ }^{1,2,3}$. \\ Gabrielle Rudolf $^{1,2,3}$ • Anne Thibault-Stoll ${ }^{1}$. Thierry Frebourg ${ }^{8,9,11}$. \\ Didier Hannequin $^{8,9,10,11,12}$ - Dominique Campion ${ }^{8,9,10,13} \cdot$ Jean-Luc Battini $^{4,5,6,7}$. \\ Marc Sitbon $^{4,5,6,7} \cdot$ Gaël Nicolas ${ }^{8,9,10,11}$
}

Received: 4 April 2016/Revised: 10 May 2016/Accepted: 10 May 2016

(c) Springer-Verlag Berlin Heidelberg 2016

\begin{abstract}
Mutations in XPRl, a gene encoding an inorganic phosphate exporter, have recently been identified in patients with primary familial brain calcification (PFBC). Using Sanger sequencing, we screened XPRI in 18 unrelated patients with PFBC and no SLC20A2, PDGFB, or $P D G F R B$ mutation. XPRI variants were tested in an in vitro physiological complementation assay and patient blood cells were assessed ex vivo for phosphate export. We identified a novel c.260T > C, p.(Leu87Pro) XPRI variant in a 41-year-old man complaining of micrographia and dysarthria and demonstrating mild parkinsonism, cerebellar
\end{abstract}

M. Anheim, U. López-Sánchez contributed equally to the work.

Electronic supplementary material The online version of this article (doi:10.1007/s00415-016-8166-4) contains supplementary material, which is available to authorized users.

Jean-Luc Battini

jean-luc.battini@igmm.cnrs.fr

$\triangle$ Marc Sitbon

marc.sitbon@igmm.cnrs.fr

$\triangle$ Gaël Nicolas

gaelnicolas@hotmail.com

1 Département de Neurologie, Hôpital de Hautepierre, CHU de Strasbourg, Strasbourg, France

2 Institut de Génétique et de Biologie Moléculaire et Cellulaire (IGBMC), INSERM-U964/CNRS-UMR7104/Université de Strasbourg, Illkirch, France

3 Fédération de Médecine Translationnelle de Strasbourg (FMTS), Université de Strasbourg, Strasbourg, France

4 Institut de Génétique Moléculaire de Montpellier, CNRS UMR5535, 1919 Route de Mende, 34293, Montpellier, France

5 Université de Montpellier, Cedex 5, 34293 Montpellier, France ataxia and executive dysfunction. Brain ${ }^{123}$ I-Ioflupane scintigraphy showed marked dopaminergic neuron loss. Peripheral blood cells from the patient exhibited decreased phosphate export. XPR1 in which we introduced the mutation was not detectable at the cell surface and did not lead to phosphate export. These results confirm that loss of XPR1-mediated phosphate export function causes PFBC, occurring in less than $8 \%$ of cases negative for the other genes, and may be responsible for parkinsonism.

Keywords Primary familial brain calcification · Idiopathic basal ganglia calcification $\cdot$ Phosphate export $\cdot$ XPR1 F Fahr disease

Laboratory of Excellence EpiGenMed, Montpellier, France

7 Laboratory of Excellence GR-Ex, Paris, France

8 Faculté de Médecine, Inserm U1079, University of Rouen, IRIB, Normandy University, 22 Boulevard Gambetta, 76183 Rouen, France

9 Normandy Centre for Genomic Medicine and Personalized Medicine, Rouen, France

10 CNR-MAJ, Rouen University Hospital, Rouen, France

11 Department of Genetics, Rouen University Hospital, Rouen, France

12 Department of Neurology, Rouen University Hospital, Rouen, France

13 Department of Research, Rouvray Psychiatric Hospital, Sotteville-Lès-Rouen, France 


\section{Introduction}

Primary familial brain calcification (PFBC) is a rare condition defined by calcification affecting at least both lenticular nuclei more severely than during uncomplicated aging [1], with no cause identified following accurate etiological assessment [2]. Calcification may also affect caudate nuclei, thalami, dentate nuclei, cerebellum, supratentorial white matter and cortical sulci. Clinical signs associated with PFBC are numerous and optional. In a French-Brazilian case series, $58 \%$ patients exhibited at least one neuropsychiatric sign [3], of which the most frequent were psychiatric signs $(76 \%$ of symptomatic patients), movement disorders (MD) $(61 \%)$ and cognitive impairment $(58 \%)$, followed by speech, gait, cerebellar syndrome, pyramidal signs, and seizures. Akinetic-hypertonic syndrome, with or without tremor, was the most common MD. Inheritance of PFBC is autosomal dominant. Four causative genes have been identified: SLC2OA2 [4], PDGFRB [5], PDGFB [6] and, most recently, XPRI [7]. $S L C 20 A 2$ encodes PiT2, an inorganic phosphate importer and we have recently shown that XPR1 [8] is the only known human phosphate exporter [9, 10]. SLC20A2 loss of function variants have been identified in a majority of cases [11], and all $4 X P R 1$ missense variants identified in 5 unrelated families were located in the SPX putative regulatory domain of XPR1, causing significantly altered XPR1-mediated phosphate export [7]. In this study, we aimed at extending these recent clinical, molecular and functional data by screening new patients and functional characterization of the variants.

\section{Methods}

\section{Patients}

Patients were included following previously described criteria [1]. Briefly, probands exhibited (1) at least bilateral lenticular calcification, (2) a total calcification score (TCS) above the age-specific threshold using our visual rating scale [1], and (3) a negative etiological assessment.

The objective of the etiological assessment was to search for other causes of brain calcification. For review see Ref. [12]. The assessment was based on clinical examination, brain MRI when available, and the exclusion of causes of non-syndromic brain calcification by the following minimal biological assessment: Phosphate and calcium levels in blood and urine, parathormone and 25-OH-vitamine D levels, and blood lactates and pyruvates. Depending on the clinical and MRI presentation, other assessments may be required to look for other causes; however, this was not the case for any of the patients included in this study.

The patients gave written consent and local ethics committee approved the study. All patients were negatively screened for $S L C 20 A 2, P D G F B$, and PDGFRB variants by Sanger sequencing and Quantitative Multiplex PCR of Short Fluorescent fragments (QMPSF) (SLC2OA2, PDGFB). XPR1 sequencing was performed by Sanger sequencing as previously described [7]. The reference transcript NM_004736.3 was used for variant nomenclature.

\section{Functional analyses}

\section{Cells}

HEK293T cells were cultured in DMEM supplemented with $10 \%$ FBS (PAN-Biotech) and non-essential amino acids. Cells were incubated at $37{ }^{\circ} \mathrm{C}$ in a $5 \% \mathrm{CO}_{2}$ and humid atmosphere. For phosphate-free experiments, cells were cultured in phosphate-free DMEM supplemented with $10 \%$ dialyzed FBS $[7,10]$.

\section{Plasmids and siRNAs}

The c.260T $>$ C, p.(Leu87Pro) mutation, thereafter referred to as L87P, was generated by recombinant PCR sitedirect mutagenesis. The HA-tagged human wild type (WT) and L87P XPR1 variant were introduced in the pCHIX [13] and pLXSN-retroviral vectors [14]. Small interfering RNAs (siRNA, Integrated DNA Technologies) targeting the human XPRI $3^{\prime} \mathrm{UTR}$ and the firefly luciferase gene were described previously [10]). For the XPR1 complementation assay, HEK293T cells grown on poly-D-lysinecoated 6-well plates were transfected with 50 pmol siRNA per well using the calcium phosphate method along with either empty or XPR1 expression vectors.

\section{Phosphate fluxes}

Phosphate uptake and efflux assays were performed in HEK293T cells as previously described [10]. Percentage of phosphate uptake was calculated as the ratio of cellular $\left[{ }^{33} \mathrm{P}\right]$ phosphate to total $\left[{ }^{33} \mathrm{P}\right]$ phosphate supplemented. Percentage of phosphate efflux was calculated as the ratio of released $\left[{ }^{33} \mathrm{P}\right]$ phosphate to total cellular $\left[{ }^{33} \mathrm{P}\right]$ phosphate. Although synchronization was arduous, we were able to perform phosphate efflux assays in peripheral blood mononuclear cells (PBMC) from some patients as previously described [7]. 


\section{Immunoblotting}

Cell extracts were separated by $12 \%$ SDS-PAGE under reducing conditions and transferred to PVDF membranes. Protein expression and transfer were monitored with antiHA tag antibodies (3F10, Roche; $1: 5000)$ or anti- $\beta$-actin (A5441, Sigma-Aldrich; 1:5000), followed by horseradish peroxidase (HRP)-conjugated anti-mouse or anti-rat antibodies (Southern Biotech; 1:5000), and visualization with Pierce ECL western blotting substrate (Thermo Scientific).

\section{Flow cytometry}

Cell surface expression of phosphate transporters was monitored with soluble ligands derived from the receptorbinding domain (RBD) of different retroviral envelope glycoproteins. Production of and binding with RBD from X-MLV (XRBD), and koala retrovirus (KoRBD), or with a soluble ligand derived from the surface unit (SU) of amphotropic-MLV (ASU), used to detect XPR1, PiT1, and PiT2, respectively were performed as previously described $[10,15]$. Briefly, $5 \times 10^{5}$ cells were resuspended in PBA (PBS with $2 \% \mathrm{FBS}$ ) containing the adequate RBD and incubated for $30 \mathrm{~min}$ at $37^{\circ} \mathrm{C}$, followed by two washes with PBA and incubation with an Alexa Fluor 488-conjugated anti-mouse IgG1 antibody (Life Technologies; 1:5000) for $20 \mathrm{~min}$ at $4{ }^{\circ} \mathrm{C}$. Cells were promptly analyzed on FACSCalibur instrument (Becton-Dickinson) and data were analyzed with the FlowJo package.

\section{Statistical analysis}

Student's $t$ test was applied using GraphPad Prism 6 software; $P$ values were as follows: $* P \leq 0.05$, ** $P \leq 0.01$, $* * * P \leq 0.001, * * * * P \leq 0.0001$.

\section{Results}

Eighteen patients with PFBC were included: 6 probands with a pedigree suggestive of an autosomal dominant inheritance and 12 sporadic cases. In one patient, we identified a novel XPRl missense variant, c.260T $>\mathrm{C}$, p.(Leu87Pro), thereafter referred as L87P, not reported in exome databases, including the ExAC 60,706 exomes (http://exac.broadinstitute.org/). The change, located on a highly conserved residue, is predicted to affect protein function by at least three programs (Mutation Taster, SIFT, Polyphen2). DNA of relatives was not available. This variant was absent from 126 French control exomes (not shown).

The patient carrying the L87P variant is a right-handed 41-year-old man, with neither previous medical history nor treatment. He was referred to our tertiary MD center because he was complaining of insidious, slowly progressive writing difficulties, slight dysarthria and concentration deficiency for 10 years. His father experienced parkinsonism as well as dysarthria and concentration deficiency, associated with calcification on CT scan before dying from cancer at age 64. The patient's paternal aunt was diagnosed with Parkinson's disease but subsequently lost to follow-up (Fig. 1). Examination of the proband revealed mild dysarthria suggestive of both parkinsonism and cerebellar dysfunction, micrography (which was not the case several years before), mild hypermetria during the finger chase test and mildly abnormal fast alternative hand movement. There was mild rigidity of right wrist (1/4) but no tremor, dystonia, chorea, or imbalance. SARA score was 3/40 [16] and UPDRS motor score was 3/108. Anxiety was noticed and neuropsychological tests showed mild executive dysfunction with mild disturbances of initiation, planification flexibility and encoding episodic memory. Brain CT scan revealed bilateral, symmetrical calcifications of lenticular and caudate nuclei, thalami, cerebellar hemispheres and vermis as well as mild sub-cortical and cortical calcifications (TCS $=46 / 80$ ) (Fig. 1) [1]. Brain scintigraphy with ${ }^{123}$ I-Ioflupane showed marked, bilateral and symmetrical dopaminergic neuron loss (Fig. 2) with a mean specific striatum uptake of 2.3 (right) and 2.2 (left) (normal values $>3.5$ ) whereas the putamen/caudate uptake ratio was 0.72 (right) and 0.71 (left) (normal values $>0.85$ ). A new examination at age 42 revealed no particular change although the patient felt better following speech and writing therapy.

In a complementation assay, we found that WT XPR1 fully complemented phosphate export inhibited by a siRNA directed against XPR1 $3^{\prime}$ UTR and that the L87P-

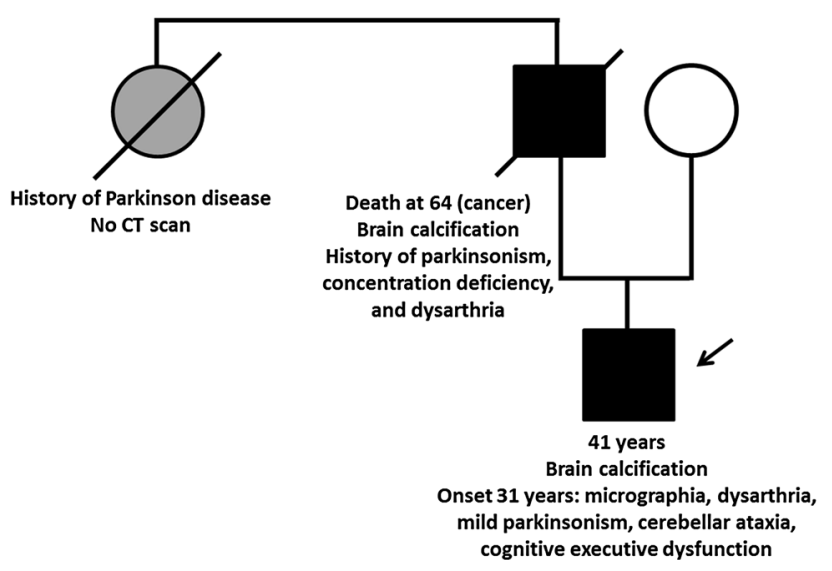

Fig. 1 Reduced pedigree of the patient carrying the novel p.(Leu87Pro) XPR1 variant. Filled symbols represent patients carrying brain calcification and the grey circle represents the paternal aunt with a history of parkinsonism but no CT scan 


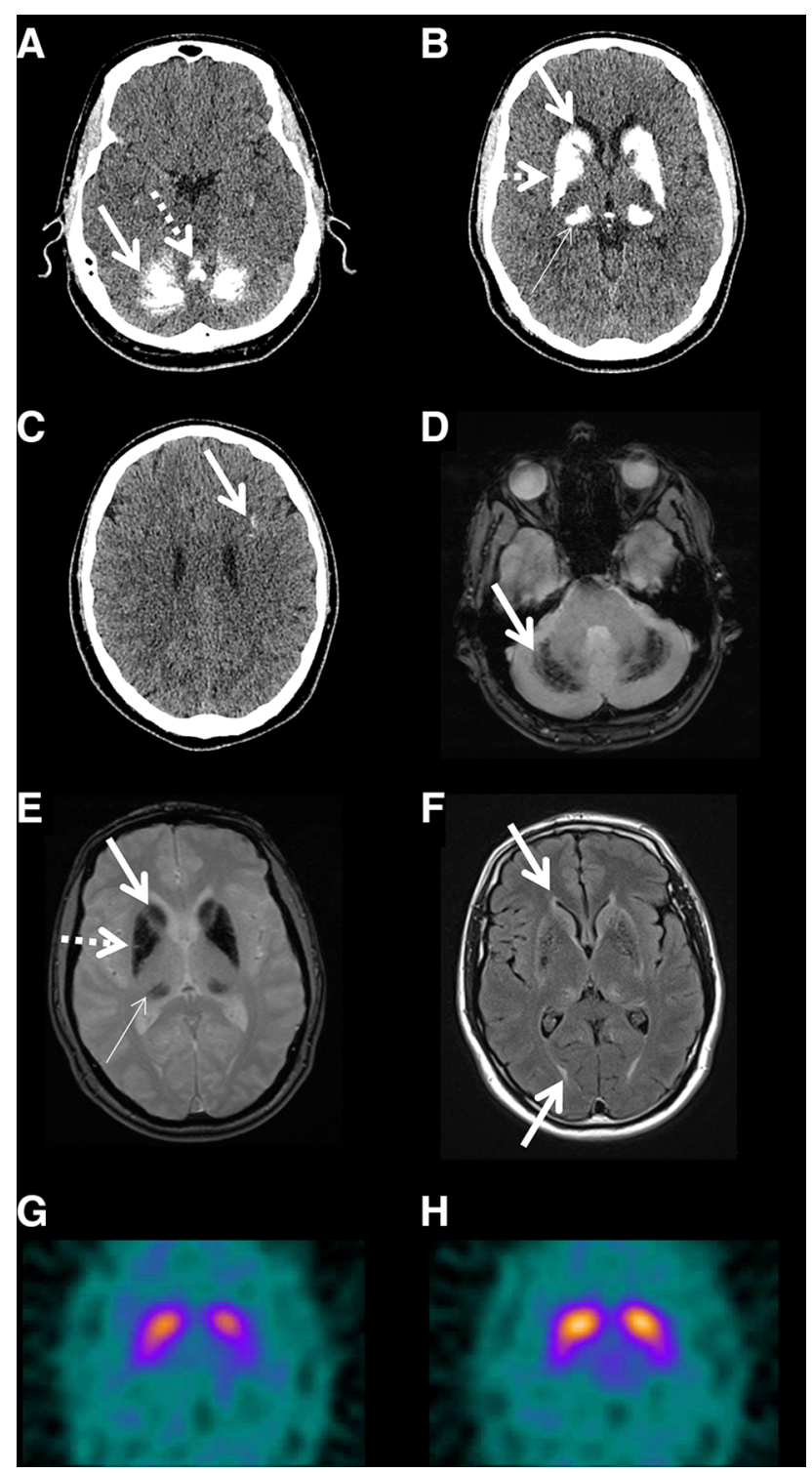

Fig. 2 Brain imaging of the patient carrying the novel p.(Leu87Pro) XPR1 variant. Calcifications appear hyperdense on CT scan $(\mathbf{a}, \mathbf{b}$, c) and hypointense on T2*-weighted MRI (d, e). They affect both cerebellar hemispheres (a, d, full arrows), the vermis (a, dotted arrow), the caudate nuclei (b, e, full arrows), the lenticular nuclei (b, e, dotted arrows), the thalami (thin arrows), cerebral white matter (not shown) and the cortex in the depth of one sulcus (c). On FLAIRweighted images, white matter hyperintensities are observed in periventricular regions and posterior cerebral white matter (f). 123Ioflupane scintigraphy axial slides $(\mathbf{g}, \mathbf{h})$ : marked, bilateral loss of nigro-striatal dopaminergic neurons with left predominance

mutated XPR1 did not (Fig. 3a), despite efficient expression, at the expected size of $82 \mathrm{Kda}$ albeit at a slightly lower level than WT XPR1 (Fig. 3b). However, the mutant was not detected at the cell surface, as monitored by flow cytometry with XRBD, the XPR1 ligand (Fig. 3c), while phosphate uptake and cell surface expression of the PiT1 and PiT2 phosphate importers remained unchanged
(Supplementary Figure). Decreased phosphate export was also observed in PBMC obtained from the L87P PFBC patient (Fig. 3d).

\section{Discussion}

This is the first report of an XPRI pathogenic variant in a PFBC patient since our primary report [7], confirming that $X P R 1$ is involved in the genetic determinism of PFBC and may be responsible for parkinsonism. XPRl mutations are scarce since within the group of 65 patients negatively screened for $S L C 20 A 2, P D G F B$, and PDGFRB, including the 18 patients reported here and the 47 patients previously reported with the same inclusion criteria [7], 4 different XPRI pathogenic variants (i.e. class 5 following ACMG recommendations [17]) in 5 families were found $(7.7 \%$, namely p.Ser136Asn, p.Leu140Pro, p.Leu145Pro, and p.Leu218Ser). Two other rare missense variants (p.Lys53Arg and p.Ile575Val) were detected in the primary report but were predicted benign or tolerated by SIFT and Polyphen 2 programs with probably no effect on XPR1 function, as already shown for the p.Lys53Arg variant [7]. These two variants are therefore considered as likely nonpathogenic (class 2 variants).

Although global correlation between calcification severity and symptomatic status has been observed in case series [1, 3, 18], no individual correlation could be inferred, as illustrated by the apparent severity of the calcifications on CT scan in our patient and the absence of marked disability even after 10 years of disease duration. In the same way, parkinsonism was mild whereas the loss of dopaminergic neurons was marked. Of note, although relatives could not be examined and DNA was not available, clinical presentation was quite homogeneous in this family since all experienced parkinsonism and that, according to the patient, his clinical picture was comparable to his father's. Brain calcification involved both lenticular, caudate nuclei, thalami, cerebellar hemispheres as well as vermis and the depth of one cortical sulcus, as observed in several but not all XPRI mutation carriers [7]. In PFBC, calcification leads to the diffuse involvement of the brain which causes potential combination of numerous unspecific signs including, beside parkinsonism, cerebellar ataxia, executive dysfunction and anxiety such as in our case, and, in other cases, dystonia, chorea, various psychiatric disturbances, pyramidal signs, seizures or migraine.

Herein, we showed that the L87P variant causes dramatic drop of both XPR1 cell surface expression and phosphate export, similar to what we previously reported in other patients harboring the p.(Leu145Pro) variant [7]. As L87P, all previously reported pathogenic variants (c.407G > A, p.(Ser136Asn); c.420T > C, p.(Leu140Pro); 


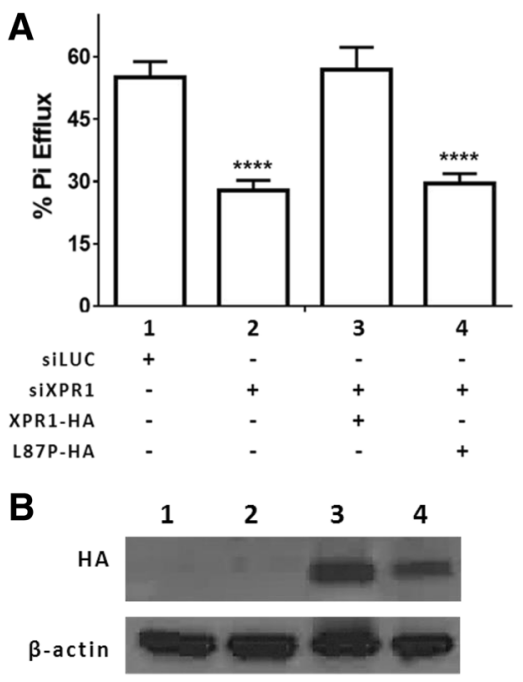

Fig. 3 Effect of the L87P mutation on XPR1 expression and phosphate efflux. a Efflux of inorganic ${ }^{33} \mathrm{P}(\mathrm{Pi})$ assay performed in HEK293T cells transfected either with siRNA directed against luciferase (siLUC), or anti XPRI siRNA (siXPR1), or siXPR1 and either an expression vector coding for HA-tagged WT XPR1 (XPR1HA) or the L87P mutant (L87P-HA) human XPR1. Results are shown as mean $\pm \mathrm{SEM}$ in a representative experiment $(n=3)$; $* * * P \leq 0.001, \quad * * * * P \leq 0.0001$, in comparison to the control (siLUC). b Immunoblot of HA-tagged XPR1-containing cell lysates probed with an anti-HA antibody (upper panel), or with an anti $\beta$ -

and c.434T $>$ C, p.(Leu145Pro) in exon 4, and c.653T $>$ C, p.(Leu218Ser) in exon 6) map to the SPX domain [7], whose exact role is not yet understood [10, 19]. We found that deletion of SPX in WT XPR1 does not alter phosphate export [10]. This and the facts that XPRI mutations found in PFBC altered XPR1-mediated phosphate efflux, including two mutations that altered presence of XPR1 at the cell surface (L87P and p.(Leu145Pro) [7]), suggests that SPX plays a regulatory role and that mutations are likely to alter interactions with factors involved in cell surface trafficking and phosphate export. XPRI mutations found in PFBC that altered phosphate export could be distinguished as forms that were not expressed at the cell surface, p.(Leu145Pro) [7] and L87P, and forms that could be efficiently detected at the cell surface. However, no obvious clinical difference could be noted between these two groups of patients, or with the carriers of $S L C 2 O A 2, P D G F B$, or $P D G F R B$ variants [3, 7].

PFBC-associated SLC2OA2 and XPR1 genes encode phosphate importer and exporter, respectively. The mechanisms underlying brain calcification with molecules transporting phosphate in opposite directions remains hypothetical. Two recent studies showed that mice with Slc20a2 haploinsufficiency presented high phosphate level in CSF, predisposing to vascular brain calcification [20, 21]. Therefore, it is tempting to propose that PiT2 and

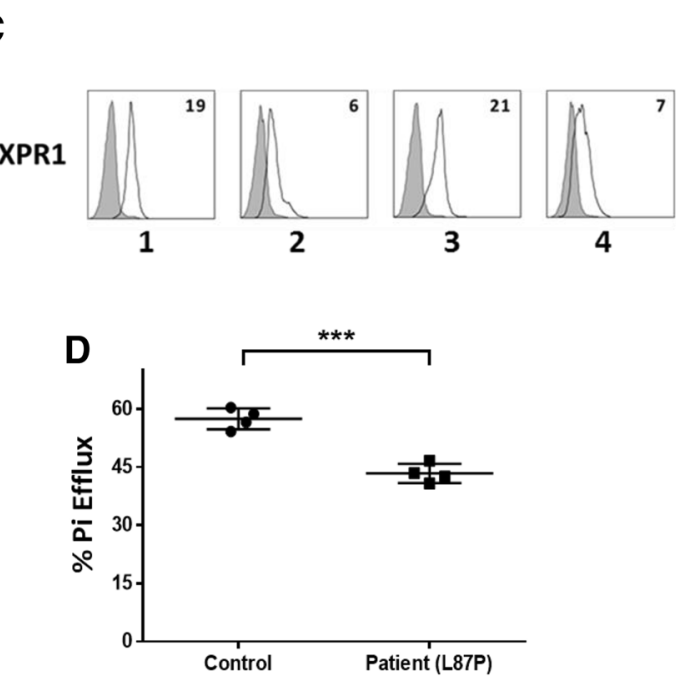

actin antibody, used as loading control (lower panel). c Cell surface detection of XPR1 with the X-MLV Env receptor-binding domain (open histograms), in HEK293T transfected cells as in a and b. Nonspecific staining with the secondary $\operatorname{IgG}$ antibody alone is shown (grey histograms). Numbers indicate the specific mean fluorescence intensity of a representative experiment $(n=3)$. d Efflux of inorganic ${ }^{33} \mathrm{P}(\mathrm{Pi})$ assay in PBMC isolated either from a healthy donor (circles) or from a PFBC patient carrying the p.(Leu87Pro) alteration (squares). Bars represent SEM $* * * P \leq 0.001$

XPR1 participate in phosphate directional transport from CSF to the blood in epithelial cells of the choroid plexus or ependyma, known for regulating ion concentrations in CSF. Finally, our study demonstrates that XPRI mutations are a scarce cause of PFBC and may be responsible for parkinsonism.

Acknowledgments We are grateful to all collaborators who sent blood samples and medical charts, and all members of our labs for constant support and helpful discussions. This work was supported by grants from the Fondation pour la Recherche Médicale (FRM) and ANR Blanc (to M.S.); Ligue Nationale contre le Cancer (Comité de 1'Hérault) and Agence Nationale de la Recherche JCJC (to J.-L.B.); U.L.-S. was supported by Labex EpiGenMed (ANR-10-LABX-12-01) and Secretaría de Ciencia, Tecnología e Innovación de la Ciudad de México (CM-102/15 SECITI 030/2016) fellowships; D.G. was supported by FRM, Institut National du Cancer (INCa), and Labex GREx (ANR-11-LABX-0051) fellowships; Labex is funded by the program 'Investissements d'Avenir' of the French National Research Agency. J.-L.B. and M.S. are supported by INSERM; G.N., D.C., T.F., and D.H. are supported by Inserm, the University Hospital of Rouen and the French CNR-MAJ.

\section{Compliance with ethical standards}

Conflicts of interest J-L.B. and M.S. are inventors on provisional a patent describing the use of ligands, including XRBD, for the analysis of human cells (PCT/EP2010/050139); M.S. is a co-founder of METAFORA-biosystems, a start-up company that focuses on metabolite transporters under physiological and pathological conditions. 


\section{References}

1. Nicolas G, Pottier C, Charbonnier C, Guyant-Marechal L, Le Ber I, Pariente J, Labauge P, Ayrignac X, Defebvre L, Maltete D, Martinaud O, Lefaucheur R, Guillin O, Wallon D, Chaumette B, Rondepierre P, Derache N, Fromager G, Schaeffer S, Krystkowiak P, Verny C, Jurici S, Sauvee M, Verin M, Lebouvier T, Rouaud O, Thauvin-Robinet C, Rousseau S, Rovelet-Lecrux A, Frebourg T, Campion D, Hannequin D (2013) Phenotypic spectrum of probable and genetically-confirmed idiopathic basal ganglia calcification. Brain 136(Pt 11):3395-3407

2. Sobrido MJ, Hopfer S, Geschwind DH (1993-2004) Familial Idiopathic Basal Ganglia Calcification. In: Pagon RA, Bird TD, Dolan CR, et al (eds) GeneReviews ${ }^{\mathrm{TM}}$ [Internet] Seattle (WA): University of Washington, Seattle

3. Nicolas G, Charbonnier C, de Lemos RR, Richard AC, Guillin O, Wallon D, Legati A, Geschwind D, Coppola G, Frebourg T, Campion D, de Oliveira JR, Hannequin D (2015) Brain calcification process and phenotypes according to age and sex: lessons from SLC20A2, PDGFB, and PDGFRB mutation carriers. Am J Med Genet B Neuropsychiatr Genet 168(7):586-594

4. Wang C, Li Y, Shi L, Ren J, Patti M, Wang T, de Oliveira JR, Sobrido MJ, Quintans B, Baquero M, Cui X, Zhang XY, Wang L, Xu H, Wang J, Yao J, Dai X, Liu J, Zhang L, Ma H, Gao Y, Ma X, Feng S, Liu M, Wang QK, Forster IC, Zhang X, Liu JY (2012) Mutations in SLC20A2 link familial idiopathic basal ganglia calcification with phosphate homeostasis. Nat Genet 44(3):254-256

5. Nicolas G, Pottier C, Maltete D, Coutant S, Rovelet-Lecrux A, Legallic S, Rousseau S, Vaschalde Y, Guyant-Marechal L, Augustin J, Martinaud O, Defebvre L, Krystkowiak P, Pariente J, Clanet M, Labauge P, Ayrignac X, Lefaucheur R, Le Ber I, Frebourg T, Hannequin D, Campion D (2013) Mutation of the PDGFRB gene as a cause of idiopathic basal ganglia calcification. Neurology 80(2):181-187

6. Keller A, Westenberger A, Sobrido MJ, Garcia-Murias M, Domingo A, Sears RL, Lemos RR, Ordonez-Ugalde A, Nicolas G, da Cunha JE, Rushing EJ, Hugelshofer M, Wurnig MC, Kaech A, Reimann R, Lohmann K, Dobricic V, Carracedo A, Petrovic I, Miyasaki JM, Abakumova I, Mae MA, Raschperger E, Zatz M, Zschiedrich K, Klepper J, Spiteri E, Prieto JM, Navas I, Preuss M, Dering C, Jankovic M, Paucar M, Svenningsson P, Saliminejad K, Khorshid HR, Novakovic I, Aguzzi A, Boss A, Le Ber I, Defer G, Hannequin D, Kostic VS, Campion D, Geschwind DH, Coppola G, Betsholtz C, Klein C, Oliveira JR (2013) Mutations in the gene encoding PDGF-B cause brain calcifications in humans and mice. Nat Genet 45(9):1077-1082

7. Legati A, Giovannini D, Nicolas G, Lopez-Sanchez U, Quintans B, Oliveira JR, Sears RL, Ramos EM, Spiteri E, Sobrido MJ, Carracedo A, Castro-Fernandez C, Cubizolle S, Fogel BL, Goizet C, Jen JC, Kirdlarp S, Lang AE, Miedzybrodzka Z, Mitarnun W, Paucar M, Paulson H, Pariente J, Richard AC, Salins NS, Simpson SA, Striano P, Svenningsson P, Tison F, Unni VK, Vanakker O, Wessels MW, Wetchaphanphesat S, Yang M, Boller F, Campion D, Hannequin D, Sitbon M, Geschwind DH, Battini JL, Coppola G (2015) Mutations in XPR1 cause primary familial brain calcification associated with altered phosphate export. Nat Genet 47(6):579-581

8. Battini JL, Rasko JE, Miller AD (1999) A human cell-surface receptor for xenotropic and polytropic murine leukemia viruses: possible role in G protein-coupled signal transduction. Proc Natl Acad Sci USA 96(4):1385-1390

9. Kavanaugh MP, Miller DG, Zhang W, Law W, Kozak SL, Kabat D, Miller AD (1994) Cell-surface receptors for gibbon ape leukemia virus and amphotropic murine retrovirus are inducible sodium-dependent phosphate symporters. Proc Natl Acad Sci USA 91(15):7071-7075

10. Giovannini D, Touhami J, Charnet P, Sitbon M, Battini JL (2013) Inorganic phosphate export by the retrovirus receptor XPR1 in metazoans. Cell Rep 3(6):1866-1873

11. Hsu SC, Sears RL, Lemos RR, Quintans B, Huang A, Spiteri E, Nevarez L, Mamah C, Zatz M, Pierce KD, Fullerton JM, Adair JC, Berner JE, Bower M, Brodaty H, Carmona O, Dobricic V, Fogel BL, Garcia-Estevez D, Goldman J, Goudreau JL, Hopfer S, Jankovic M, Jauma S, Jen JC, Kirdlarp S, Klepper J, Kostic V, Lang AE, Linglart A, Maisenbacher MK, Manyam BV, Mazzoni P, Miedzybrodzka Z, Mitarnun W, Mitchell PB, Mueller J, Novakovic I, Paucar M, Paulson H, Simpson SA, Svenningsson P, Tuite P, Vitek J, Wetchaphanphesat S, Williams C, Yang M, Schofield PR, de Oliveira JR, Sobrido MJ, Geschwind DH, Coppola G (2013) Mutations in SLC20A2 are a major cause of familial idiopathic basal ganglia calcification. Neurogenetics 14(1):11-22

12. Deng H, Zheng W, Jankovic J (2015) Genetics and molecular biology of brain calcification. Ageing Res Rev 22:20-38

13. Manel N, Kim FJ, Kinet S, Taylor N, Sitbon M, Battini JL (2003) The ubiquitous glucose transporter GLUT-1 is a receptor for HTLV. Cell 115(4):449-459

14. Miller AD, Rosman GJ (1989) Improved retroviral vectors for gene transfer and expression. Biotechniques. 7(9):980-982 4-6, 9-90

15. Petit V, Massonnet G, Maciorowski Z, Touhami J, Thuleau A, Nemati F, Laval J, Chateau-Joubert S, Servely JL, Vallerand D, Fontaine JJ, Taylor N, Battini JL, Sitbon M, Decaudin D (2013) Optimization of tumor xenograft dissociation for the profiling of cell surface markers and nutrient transporters. Lab Invest 93(5):611-621

16. Schmitz-Hubsch T, du Montcel ST, Baliko L, Berciano J, Boesch S, Depondt C, Giunti P, Globas C, Infante J, Kang JS, Kremer B, Mariotti C, Melegh B, Pandolfo M, Rakowicz M, Ribai P, Rola R, Schols L, Szymanski S, van de Warrenburg BP, Durr A, Klockgether T, Fancellu R (2006) Scale for the assessment and rating of ataxia: development of a new clinical scale. Neurology 66(11):1717-1720

17. Richards S, Aziz N, Bale S, Bick D, Das S, Gastier-Foster J, Grody WW, Hegde M, Lyon E, Spector E, Voelkerding K, Rehm HL, Committee ALQA (2015) Standards and guidelines for the interpretation of sequence variants: a joint consensus recommendation of the American College of Medical Genetics and Genomics and the Association for Molecular Pathology. Genet Med 17(5):405-424

18. Manyam BV, Walters AS, Narla KR (2001) Bilateral striopallidodentate calcinosis: clinical characteristics of patients seen in a registry. Mov Disord 16(2):258-264

19. Vaughan AE, Mendoza R, Aranda R, Battini JL, Miller AD (2012) Xpr1 is an atypical G-protein-coupled receptor that mediates xenotropic and polytropic murine retrovirus neurotoxicity. J Virol 86(3):1661-1669

20. Jensen N, Schroder HD, Hejbol EK, Fuchtbauer EM, de Oliveira JR, Pedersen L (2013) Loss of function of slc20a2 associated with familial idiopathic Basal Ganglia calcification in humans causes brain calcifications in mice. $\mathrm{J}$ Mol Neurosci 51(3):994-999

21. Wallingford MC, Chia J, Leaf EM, Borgeia S, Chavkin NW, Sawangmake C, Marro K, Cox TC, Speer MY, Giachelli CM (2016) SLC20A2 deficiency in mice leads to elevated phosphate levels in cerbrospinal fluid and glymphatic pathway-associated arteriolar calcification, and recapitulates human idiopathic basal ganglia calcification. Brain Pathol. doi:10.1111/bpa.12362 